Christiane Rochefort, Journal pré-posthume possible, édition établie par Ned Burgess et Catherine Viollet, Paris, Éditions iXe, 2015, 283 p.

\title{
Véronique Montémont
}

\section{(2) OpenEdition}

Journals

Édition électronique

URL : https://journals.openedition.org/genesis/1353

DOI : 10.4000/genesis. 1353

ISSN : 2268-1590

Éditeur :

Presses universitaires de Paris Sorbonne (PUPS), Société internationale de génétique artistique littéraire et scientifique (SIGALES)

\section{Édition imprimée}

Date de publication : 9 mai 2016

Pagination : 195-197

ISBN : 9791023105315

ISSN : $1167-5101$

\section{Référence électronique}

Véronique Montémont, «Christiane Rochefort, Journal pré-posthume possible, édition établie par Ned Burgess et Catherine Viollet, Paris, Éditions iXe, 2015, 283 p. », Genesis [En ligne], 42 | 2016, mis en ligne le 03 novembre 2016, consulté le 17 janvier 2023. URL : http://journals.openedition.org/genesis/ 1353 ; DOI : https://doi.org/10.4000/genesis.1353 
des vingt romans ou récits qu'il comprend. Butcher leur consacre à chacun un chapitre identifié par le titre d'origine (versus le titre entériné par l'édition Hetzel), et chapeautés par une synthèse de la chronogenèse. Mais l'étude n'étend pas son champ à toute la période de supervision de Hetzel père (1862-1886) : elle compose une sélection en fonction soit de la mémoire collective (célébrité, postérité des textes), soit de préférences personnelles (textes révélateurs des tensions entre auteur et éditeur?), à quoi s'ajoutent les opus intéressants pour la période d'avant la rencontre avec l'éditeur, soit 1859-1879. C'est donc un corpus sélectif qui éclaire autant par ses choix qu'il étonne par ses rejets!

Lorsqu' on prend connaissance des recherches effectuées sur le corpus manuscrit par l'auteur de Jules Verne inédit, une double position se dégage qui tient à une façon très singulière de mêler distance critique et énonciation subjective. Tout d'abord, c'est avec un intérêt marqué que le lecteur pénètre dans la fabrique de l'œuvre, dans le processus d'écriture de Jules Verne écrivain, que l'investigation concerne le scénarique, le documentaire ou le rédactionnel suivant la disponibilité des traces écrites : mises au net, brouillons, scénarios... Sans doute tout n'est pas complètement nouveau pour le chercheur familiarisé avec l'univers des manuscrits comme en témoigne la bibliographie de cet ouvrage. En suivant pas à pas le repérage des variantes, se révèle le palimpseste des couches textuelles, par ratures, additions, réécritures successives, se dessinent les logiques de l'écrivain en négociation perpétuelle avec les contraintes ou influences de l'éditeur qui joue de sa position dominante d'agent littéraire.

Le principe de l'investigation des manuscrits consiste en effet à développer une argumentation à l'encontre de l'éditeur Pierre-Jules Hetzel : une argumentation sans concession, dans la lignée des critiques développées naguère par Olivier Dumas 2 . Sur la question de la tutelle éditoriale, le propos revient à incriminer le vague des croyances partagées par les commentaires sur l'interventionnisme de P.-J. Hetzel et à présenter en regard une véritable enquête sur les interférences consécutives à la lecture des épreuves où Jules Verne se confronte constamment aux commentaires et recommandations : c'est ce que William Butcher appelle une étude «au pied de la lettre». Dans cet ordre d'idées, il assimile l'éditeur à un «nègre» à l'influence néfaste, et recourt à des procédés de dévalorisation parfois caricaturaux par l'emploi d'un lexique de plus en plus critique ( invraisemblance», «absurdités ») et d'une rhétorique de la mutilation ( «excisé», «châtré», «éviscéré»). Ces procédés renforcent un discours du pro et contra mais selon un manichéisme qui réévalue Jules Verne à proportion des jugements négatifs sur son éditeur : par cette réévaluation se justifie la (fausse) évidence d'une foi infaillible dans le jugement littéraire de l'auteur sur sa propre œuvre. En conclusion, il faudrait considérer que le texte de Jules Verne à établir, c'est ce texte «inédit» qui aurait échappé à la corruption de l'interventionnisme de P.-J. Hetzel, pour garder l'allusion à Rousseau !

Un profil est peu à peu dressé de P.-J. Hetzel, statufié en homme d'édition pour la jeunesse conformiste et en écrivain de littérature populaire médiocre. Au terme de ce parcours biaisé des relations entre auteur et éditeur, la conclusion soulève la question éditoriale. Butcher avance une série de propositions pour les éditions futures de Jules Verne (sur la paternité des romans, sur la fidélité du texte, sur l'accès aux variantes significatives), mais il laisse en suspens la question de la publication d'un «état antérieur» à l'intervention de P.-J. Hetzel pour les romans les plus célèbres qu'il appelle de ses vœux, même s'il ne tranche pas entre les différents types d'édition possibles. Il insiste in fine sur la question de l'authenticité du texte, selon un argumentaire discutable : parler de «meilleure version» présuppose, au regard de l'orientation de l'ouvrage ici commenté, un état du texte qui s'abstrairait complètement de l'interventionnisme, de l'expertise ou de la censure de P.-J. Hetzel en fonction d'un seul guide : «l'intention probable de l'auteur». Opération à hauts risques pour le textologue : selon quels critères d'analyse? Avec quelles méthodes? Avec quelles limites?

Christiane Rochefort, Journal pré-posthume possible, édition établie par Ned Burgess et Catherine Viollet, Paris, Éditions iXe, 2015, 283 p.

\section{Compte rendu \\ par Véronique Montémont*}

\section{Sur le pied de guerre}

Christiane Rochefort, auteure de romans qui marquèrent une génération, comme Les Petits Enfants du siècle (1961) ou Stances à Sophie (1963), récompensée par le prix Médicis pour La Porte du fond (1998), fut un écrivain à la fois exigeant et populaire. Elle excellait à dépeindre, dans une langue à la fois ironique et mordante, l'hypocrisie des conventions sociales. Elle a également laissé une autobiographie, Ma vie revue et corrigée par l'auteur (1978), des méditations mêlant aphorismes, prose et poésie (Le Monde est comme deux chevaux, 1984) et des réflexions sur son travail d'écrivain (C'est bizarre l'écriture, 1970). Celle qui fut, par ailleurs, longtemps attachée de presse du festival de Cannes, avait fait le choix de vivre de sa plume. Entre 1986 et 1993, à la fin d'une vie presque entièrement consacrée à l'écriture, elle a tenu un journal et confié à son amie Misha Garrigue Burgess le soin de l'éditer après sa mort. Catherine Viollet, qui a accompagné dès le début cette entreprise éditoriale, l'a poursuivie au décès de Misha Garrigue et

2. Président de la Société Jules Verne pendant une quarantaine d'années (1969-2013), Olivier Dumas s'est investi dans les recherches sur la correspondance et les manuscrits de Jules Verne, ce qui l'a conduit à adopter des positions offensives à l'égard de l'éditeur Hetzel, père ou fils, au nom de la réhabilitation à tout prix de l'œuvre de Jules Verne (publication des posthumes notamment)

* Université de Lorraine/ATILF 
a achevé l'édition du livre avec l'aide de Ned Burgess. Comme l'explique la préface, la publication de ce texte est en elle-même une aventure génétique. En effet, Christiane Rochefort ne tenait pas son journal sous une forme continue, mais sur « un ensemble de feuilles manuscrites de grand et de petit format», regroupées dans cinq dossiers parallèles. Le titre retenu pour l'édition du Journal pré-posthume possible est celui de l'un d'entre eux, aux côtés de «Journal intermittent occasionnel», «Journal intermittent baladeur posthume», «Journal bribes à examiner» ou «Broutilles». Pas de chronique au long cours, donc, mais des notations d'écrivain, dont leur auteur, de toute évidence, avait conscience qu'elles étaient la matière d'un texte potentiel.

Misha Garrigue, Catherine Viollet et Ned Burgess ont choisi de reconstruire le journal en interclassant les dossiers, de manière à retrouver un ordre chronologique essentiel à sa compréhension. Quand elle commence son journal, Christiane Rochefort est en effet en pleine écriture de La Porte du fond. Les premières notations nous plongent dans le laboratoire d'un écrivain qui s'attache à décrire le processus complexe de la naissance d'un livre. D'abord se placer en position d'attente, laisser venir ce que l'auteure appelle des «départs in-concertés », thèmes ou amorces d'intrigues. Ensuite, «solliciter», «presser» ce matériau initial pour en faire « dégorger une essence» (28 février 1986). Chose rare, Rochefort reconnaît sans fard sa facilité à mettre en route un récit et sait avouer quand c'est «bon»: «Comme s'il y avait devant moi, écrit-elle, un grand réservoir [...] et je n'ai qu'à attendre, la main ouverte. Ça va tomber» (ibid.). La véritable bataille, c'est plus tard, avec la langue, qu'elle la livre. Quelques années après, elle revient sur cette question des débuts, en des termes explicitement militaires :

Il faut faire une percée. Trouver l'endroit. [...]

Un jour, rajouter un truc qui a l'air d'un tournant, d'aller voir ailleurs (si j'y suis). Barrer, rayer. Attendre. [...]
Ou alors, quelque chose pointe. Montre son nez dans une direction imprévue. Autre. Sort du banal réalisme, de colère ou quoi que ce soit. [...]

Alors, la percée. Sur le pied de guerre. Avancer les troupes. SI ça va quelque part, insister. Forcer (juillet 1992).

L'écriture de La Porte du fond, qui traite de l'inceste, pose de multiples problèmes. Le projet, avec ses difficultés énonciatives («Essayer d'être en même temps dans le Je-Moi et dans le Je-Autre », 9 mars 1986) conduit l'écrivain à s'interroger sur l'authenticité de sa relation au fait narré, à questionner la distance entre vie et littérature : «Est-ce qu'on peut re-créer une réalité qui a eu lieu et qui n'est plus? (Où il n'y a plus l'émotion) Je crois qu'il y en a qui l'ont fait, mais pour l'instant je ne sais plus» (9 mars 1986). L'évocation de la genèse difficultueuse du livre lève au passage le voile sur un autre aspect de la «méthode Rochefort» : le travail collectif, dont ses lecteurs avaient déjà eu un avantgoût dans Ma vie revue et corrigée par l'auteur. Non seulement l'écrivain parle de ses textes à ses amis proches, tenant avec eux ce qu'elle appelle des "conférences littéraires », mais elle leur en fait de surcroît la lecture à haute voix, avant d'intégrer au fur et à mesure leurs avis et leurs suggestions. Leur regard lui est essentiel :

Misha avec $1 \mathrm{~h} 1 \frac{1}{2}$ d'avance mais du tellement bon travail sur mes trucs $=$ Misha est JUSTE, voilà. Pas indulgente, juste-juste, au petit poil. Vraiment travaillé sur le détail poèmes et textes. Et elle en a trouvé des «très beaux » à ma surprise. Me renforce (7 novembre, midi).

Amos Kenan est un autre de ses complices particulièrement chers, avec qui parler du texte en cours, durant les «riches heures Kenan-Rochefort» (7 novembre 1991). L'amitié, en effet, prend une place cardinale dans la vie d'une femme qu'on découvre assez solitaire : c'est pourquoi la diariste ne manque jamais de noter qui lui a rendu visite et tout le plaisir qu'elle en a retiré. L'entourage amical devient d'autant plus nécessaire que la maladie gagne du terrain : atteinte, à partir du début des années quatrevingt, d'une ostéoporose aiguë, qui limite son autonomie physique et la fait beaucoup souffrir, Christiane Rochefort se sert aussi de son journal pour élaborer des stratégies d'écriture. Les notes décrivent le minutage des journées, étape par étape, pour résister à la fatigue et s'imposer plusieurs heures de travail quotidien, à placer là où on en a la force ( $« \mathrm{Au}$ lever, sans manger ni me laver, à mes papiers. Jusqu'à ce que je crève de faim», 11 mai 1991). Le texte consigne les luttes minuscules contre la cigarette l'auteure est atteinte d'une thrombose rétinienne -, les habitudes devenues nocives ( « Rien écrire debout devant la table, même pense-bête $=$ assieds-toi »), les fractures et autres aléas de «[s]a vie osseuse» (26 décembre 1989). On mesure, en lisant ces programmes plusieurs fois reformulés, combien cette lutte épuise la diariste : le 25 décembre 1989, elle s'avoue - ce qui est rare «fragile. Fatiguée. Incertaine».

Mais Rochefort est une femme combative. Si la révolte qu'elle porte chevillée au corps n'a plus que le plan rhétorique où s'exercer, son journal fait malgré tout état d'indignations récurrentes. Les cibles en sont nombreuses : les promoteurs immobiliers ( $«$ Tout ce que vous touchez, ça meurt », 2 mai 1986), les exégètes musicaux qui veulent «faire passer Mozart par [leur] trou d'cul» (11 juillet 1986), les universitaires dont elle brocarde l'art de manipuler «le langage pour parler du langage qui parle du langage» (mai 1986); enfin l'administration, avec laquelle elle entretient des relations exécrables. L'actualité mondiale la consterne, surtout le bellicisme d'un Ronald Reagan décrit par elle comme un «crétin gâteux ». En juin 1990, dans un élan de pessimisme, elle écrit : «Bon dieu mais qu'est-ce qui se passe avec cette malheureuse planète!»

Mais ni cette colère, ni l'âge, ni la maladie n'éteignent le goût de vivre, les désirs (y compris d'amour) et les émerveillements. Se dessinent en creux, dans les notations de la diariste sur ses lectures, la cartographie des auteurs qui ont compté 
pour elle, et sont pour certains de quasimodèles : Virginia Woolf ( Je ne sais pas faire comme Virginia », 1986), Iris Murdoch, que Rochefort lit assidûment et chez qui elle trouve "la littérature. L'art. [...] la présence» (22 mars 1986), Primo Levi, Romain Gary. L'auteure ne se sent, en revanche, aucune affinité avec le nouveau roman et exécute Robbe-Grillet d'une phrase : «des descriptions qui ne soient rien de plus que ce qu'elles disent, on peut s'en passer» (1986). Elle remarque qu'elle n'a «aucun regret de ne pas avoir été André Gide» (et ajoute «Ni lui moi, probablement», juillet 1991). C'est néanmoins un type de littérature qu'elle estime formatée pour séduire qui déchaîne ses foudres : en font les frais «le petit Lévy (B.H., pas Raymond : Raymond est un écrivain », mars 1986), Régine Deforges, dont La Bicyclette bleue est qualifiée de «Harlequin beau linge», et Michel Horst, prix Goncourt 1986, «le plus mauvais livre de l'année». Plus que du mépris, on perçoit dans ces lignes l'expression cinglante de la déception de l'écrivain, qui voit ses confrères et consœurs galvauder l'idée sans concession qu'elle se fait de la littérature.

Le Journal pré-posthume possible, témoignage de la condition d'un écrivain en cours de création ( «Les jours où je n'écris pas, où je n'essaye pas, je me sens inutile sur la terre», écrit son auteur le 6 mars 1986), se révèle enfin un texte d'une variété littéraire remarquable, où se côtoient courts poèmes, notations en prose, listes et aphorismes - dont l'humour lapidaire n'est pas sans rappeler par endroits celui
d'Erik Satie. Les éditeurs ont pris le soin d'enrichir ces pages transcrites avec des dessins, photographies et croquis, ceux de Rochefort et de ses amis; ils y ont ajouté des extraits manuscrits et ont complété le volume avec un cahier photographique tiré de l'album personnel de la diariste. Cet élégant travail de mise en pages, tout d'intelligence et de sensibilité, contribue à placer le lecteur au plus proche de la matérialité de l'écriture du texte, et à lui donner un aperçu des talents pluriels de son auteure. Mais il fait aussi du journal une fenêtre ouverte sur une personnalité atypique et attachante, celle d'une femme qui écrivait en 1986 : «Mon courage est aussi violent que ma peur». 